\title{
Tin Dioxide Thin-Film-Based Ethanol Sensor Prepared by Spray Pyrolysis
}

\author{
Rajarshi Krishna Nath and Siddhartha Sankar Nath* \\ Department of Physics, National Institute of Technology, Silchar-788010, Assam, India
}

(Received June 23, 2008; accepted August 22, 2008)

Key words: tin dioxide, spray pyrolysis, blue shift, ethanol sensor

Thin films of tin dioxide $\left(\mathrm{SnO}_{2}\right)$ were grown on glass substrate by chemical spray pyrolysis. The optical band gap of the films was studied and a blue shift was observed from 3.62 to $3.82 \mathrm{eV}$ as the thickness of the film decreased from 1035 to $233 \mathrm{~nm}$. The sensing characteristics of these films were studied at various temperatures and concentrations of ethanol. It was observed that the sensitivity increased with the working temperature. A maximum sensitivity of $98.71 \%$ to $500 \mathrm{ppm}$ ethanol was observed at $548 \mathrm{~K}$. A rapid increase in response at lower concentrations and a gradual increase in response at higher concentrations of ethanol were observed. Moreover, the films showed fast response and recovery times at higher working temperatures.

\section{Introduction}

Transparent conductive oxides (TCOs) are widely used in a variety of applications such as optoelectronics, flat panel displays (FPDs), solar cells, gas sensors, and photocatalysts. Among the existing $\mathrm{TCOs}, \mathrm{SnO}_{2}$ thin films are attractive owing to their unique physical properties such as high electrical conductivity, high transparency in the visible part of the spectrum, and high reflectivity in the infrared region. In particular, $\mathrm{SnO}_{2}$ films are stable at high temperatures, have excellent resistance to strong acids and bases at room temperature, are resistant to mechanical wear, and have very good adhesion to many substrates. ${ }^{(1)}$ Furthermore, $\mathrm{SnO}_{2}$ films are more stable than the other TCO films such as zinc oxide $(\mathrm{ZnO})$ and Sn-doped $\mathrm{In}_{2} \mathrm{O}_{3}$ (ITO). ${ }^{(1)}$ Moreover, they have a lower material cost. Scientific and technological interests demand the synthesis of ultrafine tin dioxide thin films owing to their superior physical and chemical properties and their uses as catalysts for the oxidation of organic compounds and gas sensors, rechargeable Li batteries, and optical electronic devices. ${ }^{(1)}$

Undoped and doped $\mathrm{SnO}_{2}$ thin films have been fabricated and investigated by several researchers for the detection of toxic pollutant gases, combustible gases, and organic vapors. ${ }^{(2-5)}$ Sahay et al. ${ }^{(6,7)}$ have studied the sprayed $\mathrm{ZnO}$ thin film for the detection of

"Corresponding author: e-mail: nathss1@rediffmail.com 
acetone and ethanol vapors. Shamala et al. have examined the LPG-sensing properties of undoped and antimony-doped tin oxide films prepared by spray pyrolysis and electron beam evaporation. ${ }^{(8)}$ Senguttuvan et al.$^{(9)}$ have studied the gas-sensing properties of leaddoped tin oxide thick film. Hellegouarc'h et al. have prepared the $\mathrm{SnO}_{2}$ thin film by PECVD and studied its response towards ethanol vapors. ${ }^{(10)}$

A large number of techniques have been used for the preparation of tin dioxide films. These include rf sputtering, dc glow discharge methods, reactive sputtering, nonreactive sputtering, ion-beam sputtering, electron beam evaporation, spray hydrolysis, chemical vapor deposition (CVD), and vacuum evaporation. Among these techniques, spray pyrolysis is easy, simple, cheap and suitable for large-scale production. In this study we determined the optical, electrical, and sensing properties of spray-deposited $\mathrm{SnO}_{2}$ thin film, which is completely our original work and technologically very significant.

\section{Experimental Details}

The glass substrates (microscopic slides) prepared in $37.5 \times 12.5 \times 1.35 \mathrm{~mm}^{3}$ dimensions were subsequently cleaned in freshly prepared chromic acids, followed by washing with detergent solution and distilled water. The cleaned substrates were placed on a hot plate maintained at a constant temperature of $410 \pm 15^{\circ} \mathrm{C}$. The precursor solution used was of $0.1 \mathrm{M}$ concentration of high-purity stannic chloride pentahydrate $\left(\mathrm{SnCl}_{4}{ }^{\circ}\right.$ $5 \mathrm{H}_{2} \mathrm{O}$ ) dissolved in distilled water. The atomization of the solution into a spray of fine droplets was carried out by the spray nozzle and compressed air was used as the carrier gas. A schematic of the typical spray pyrolysis setup fabricated in our laboratory is shown in Fig. 1. The various process parameters used in the film preparation are listed in Table 1. The substrate temperature was monitored using a chromel-alumel thermocouple together with a Motwane digital multimeter (Model: 454). During the optimization of the process parameters, the substrate temperature was found to be the most important parameter in the film preparation for gas-sensing applications. The thicknesses of the films were measured by the weight difference method using an electronic high-precision balance (Citizen, Model CY 204).

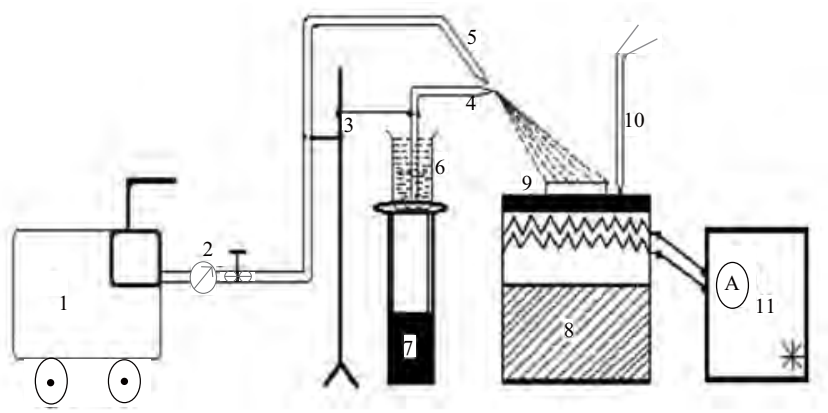

Fig. 1. Schematic representation of the spray system. (1) Air compressor, (2) pressure regulator and gauge, (3) iron stand, (4) spray nozzle, (5) air output nozzle, (6) spray solution container (beaker), (7) beaker stand, (8) block furnace, (9) substrate, (10) thermocouple, and (11) temperature control unit. 
Table 1

Process parameters for the deposition of $\mathrm{SnO}_{2}$ thin films.

\begin{tabular}{ll}
\hline Spray parameters & Optimum value/item \\
\hline Nozzle & Glass \\
Nozzle-substrate distance & $45 \mathrm{~cm}$ \\
Solution concentration & $0.1 \mathrm{M}$ \\
Solvent & Distilled water \\
Solution flow rate & $6 \mathrm{ml} / \mathrm{min}$ \\
Carrier gass & Compressed air \\
Gas pressure & $4 \mathrm{~kg} / \mathrm{cm}^{2}$ \\
Substrate temperature & $(410 \pm 15)^{\circ} \mathrm{C}$ \\
\hline
\end{tabular}

The as-deposited films were characterized by optical and electrical techniques. The optical absorption spectra of the films of different thicknesses were obtained in the ultraviolet (UV)/visible/near infrared (NIR) region up to $1100 \mathrm{~nm}$ using a Perkin Elmer UV-VIS spectrophotometer (Model: Lamda 35). The measurements were carried out in the wavelength-scanning mode under the following parametric conditions:

Incidence-Normal

Temperature of the sample-Room temperature

Reference w.r.t the sample-Uncoated glass slide

High-conducting silver paste was used to make the ohmic contacts on both ends of the films to carry out electrical measurements. The film was mounted on a custom-build two-probe assembly placed in a silica tube, which was inserted coaxially inside a tubular furnace. The electrical resistance of the film was measured before and after exposure to ethanol vapor using a Keithley System electrometer (Model: 6514). The sensing characteristics of the film at different concentrations of ethanol were studied at different operating temperatures in the range of 498-623 K. To study the sensing properties of the film, a required volume of ethanol was injected into the closed silica tube maintained at various temperatures, and subsequently, the decrease in film resistance was monitored until it became constant. Finally, both ends of the tube were opened and the film resistance was allowed to return to the initial value in air.

\section{Results and Discussion}

When aerosol droplets arrive close to the heated substrate, a pyrolytic (endothermic) reaction

$$
\mathrm{SnCl}_{4}+2 \mathrm{H}_{2} \mathrm{O} \stackrel{\text { heat }}{\longrightarrow} \mathrm{SnO}_{2}+4 \mathrm{HCl}
$$

occurs and highly adherent films of undoped $\mathrm{SnO}_{2}$ are formed on the substrate surface. The films were found to be clear and almost transparent. In this work, the thicknesses of the films were found to be within the range of $200-1100 \mathrm{~nm}$. 


\subsection{Optical studies}

The optical absorption measurements were carried out on a large number of samples of various thicknesses. Figure 2 shows some representative optical absorbance curves of $\mathrm{SnO}_{2}$ thin films as a function of wavelength for different thickness, viz, 233, 540, and $1035 \mathrm{~nm}$. These spectra revealed that films grown under the same parametric conditions have low absorbance in the visible/NIR region while the absorbance is high in the ultraviolet region. The energy band gap of $\mathrm{SnO}_{2}$ film can be roughly determined from the absorption spectra. For this, the fundamental absorption coefficient $(\alpha)$ was evaluated using Lambert's law as follows ${ }^{(11)}$

$$
\operatorname{Ln}\left(I_{0} / I\right)=2.303 A=\alpha d
$$

where $I_{0}$ and $I$ are the intensities of incident light and transmitted light, respectively, $A$ is the optical absorbance, and $d$ is the film thickness.

According to the Tauc model,

$$
\alpha h v=\mathrm{D}\left(h v-E_{\mathrm{g}}\right)^{m}
$$

where $h v$ is the photon energy, $E_{\mathrm{g}}$ is the optical band gap, D is a constant, and $m$ is an exponent that can have the values $1 / 2,3 / 2,2$, or 3 depending on the nature of the electronic transition. By replacing $n=1 / m$ and plotting an $(\alpha h v)^{n}$ versus $h v$ graph and extrapolating the linear portion of the graph to the photon energy axis, the energy gap can be derived. For a direct transition, $n=2$ or $2 / 3$ depending on whether the transition is allowed or forbidden in the quantum mechanical sense. Similarly, $n=1 / 2$ or $1 / 3$ for indirectly allowed or forbidden transition, respectively. In this work, $n=2$ was found to be the most suitable, since it gives the best linear curve in the band-edge region, which indicates a directly allowed transition in the $\mathrm{SnO}_{2}$ film material.

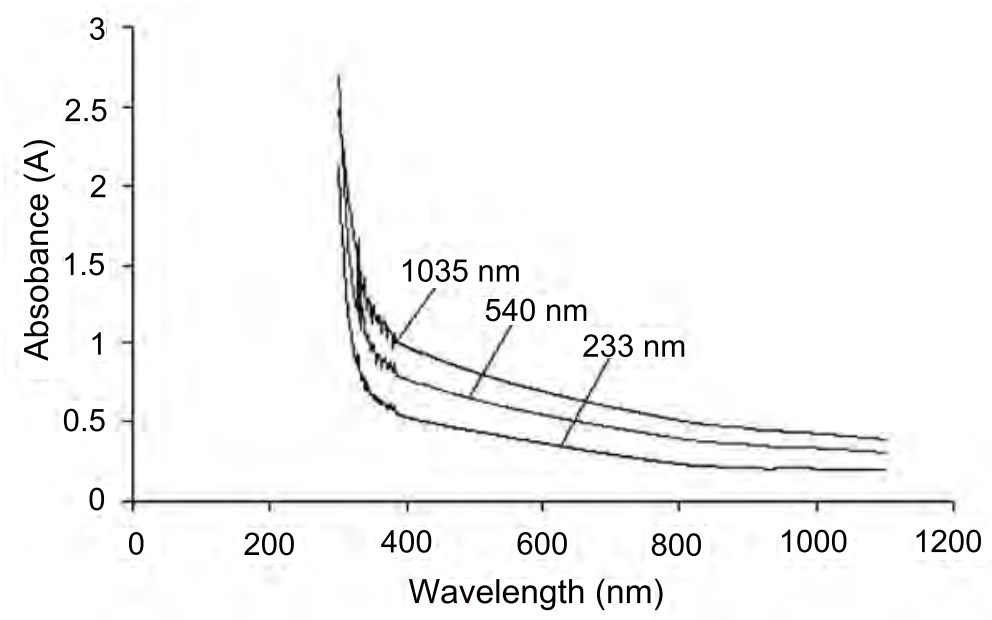

Fig. 2. Absorbance curves for the sprayed $\mathrm{SnO}_{2}$ films of various thicknesses. 
Figure 3 shows the relationship between $(\alpha h v)^{2}$ and $h v$ for $\mathrm{SnO}_{2}$ thin films of various thicknesses. The optical band gap values for different thicknesses $(233,540$, and 1035 $\mathrm{nm}$ ), calculated by the above-mentioned method are summarized in Table 2. It was observed that as the film thickness decreases from 1035 to $233 \mathrm{~nm}$, the optical band gap blue-shifted from 3.62 to $3.82 \mathrm{eV}$. In general, the variation of the band gap due to thickness can arise owing to one or a combined effect of the following causes:(12) (i) a large density of dislocations, (ii) a quantum size effect, and (iii) a change in barrier height owing to the change in grain size in polycrystalline films. However, in the present case, the first cause seems reasonable owing to the possibility of structural defects in the films, which arose at the time of preparation, and because the thicknesses of the films are quite large, the quantum size effect can be ruled out completely. A similar blue shift phenomenon of optical band gap was also observed in $\mathrm{ZnO}$ thin films in our earlier work . ${ }^{(1)}$ The dependence of optical band gap on film thickness is shown in Fig.4.

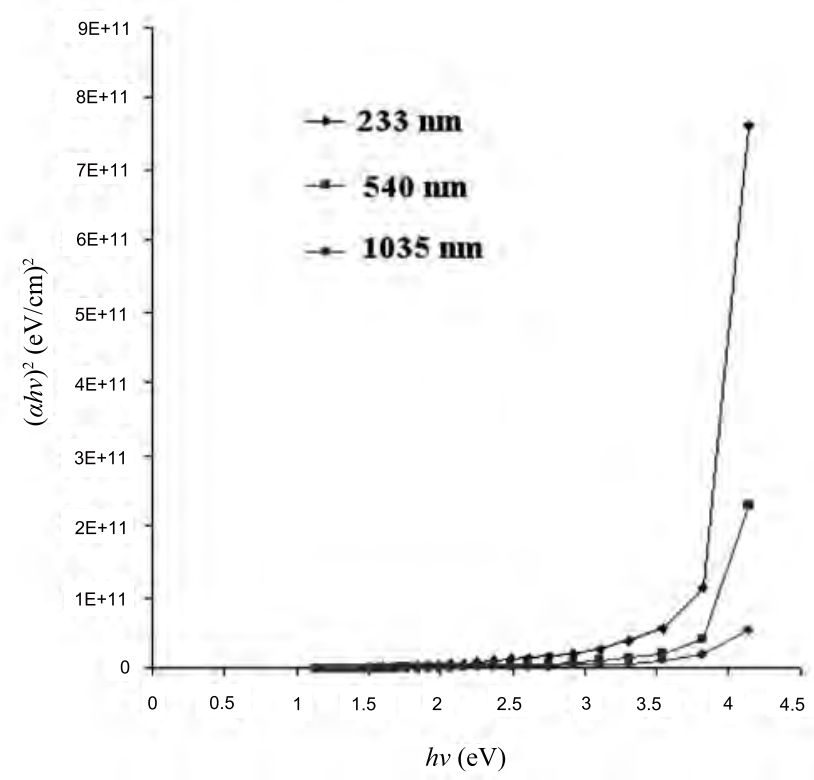

Fig. 3. Plots of $(\alpha h v)^{2}$ versus $h v$ curves of undoped $\mathrm{SnO}_{2}$ films of various thicknesses.

Table 2

Estimated optical band gap of $\mathrm{SnO}_{2}$ films of various thicknesses.

\begin{tabular}{lcc}
\hline SI no. & Film thickness $(\mathrm{nm})$ & Estimated optical band gap $(\mathrm{eV})$ \\
\hline 1 & 233 & 3.82 \\
2 & 540 & 3.71 \\
3 & 1035 & 3.62 \\
\hline
\end{tabular}




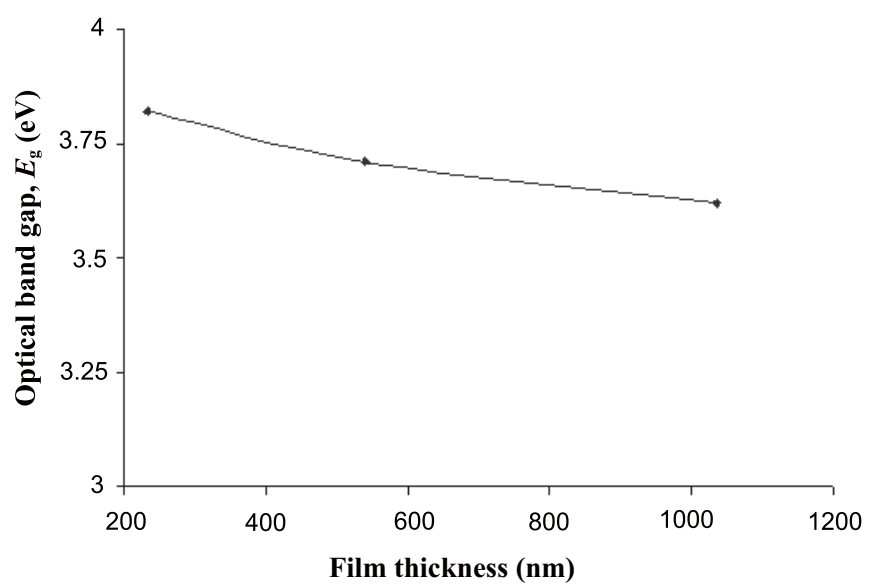

Fig. 4. Thickness dependence of optical band gap $\left(E_{\mathrm{g}}\right)$.

\subsection{Resistivity studies}

The dependence of the resistivity of the $\mathrm{SnO}_{2}$ thin film on temperature is shown in Fig. 5. As evident from the figure, the film shows four-region behavior as reported by Sahay et al. for $\mathrm{ZnO}$ film. ${ }^{(13)}$ In region $\mathrm{I}$, the decrease in resistivity is due to the thermal excitation of electrons in the conduction band. The sharp increase in resistivity in region II is attributed to the vigorous oxygen adsorption on the film surface. In region III, the resistivity is not markedly affected by the temperature change. This could be due to the equilibrium achieved between the two competing processes of thermal excitation of electrons and oxygen adsorption. Finally, the resistivity in region IV decreases again. This is attributed to the dominant thermal excitation of electrons and the desorption of oxygen species.

\subsection{Ethanol-sensing properties}

When an $n$-type semiconductor material is used as a sensor either in thin or thick film, it is basically found to possess two types of sensing effect.

(a) At first, atmospheric oxygen is adsorbed on the sensor surface, and after removing the carrier from the conduction band of the $n$-type semiconductor, it becomes an $\mathrm{O}^{2-}$ or $\mathrm{O}^{-}$species.

(b) Reducing agents such as ethanol, methanol, and acetone react with the chemi-adsorbed oxygen, thereby the gaseous species reinjects the carrier, and hence, decreases the sensor resistance.

Given that the resistance of the film decreases upon exposure to ethanol vapor, because of its reducing nature, the sensitivity is defined as

$$
S=\frac{\left(R_{\mathrm{a}}-R_{\mathrm{g}}\right)}{R_{\mathrm{a}}} \times 100 \%,
$$

where $R_{\mathrm{a}}=$ resistance of the film in air, and $R_{\mathrm{g}}=$ resistance of the film upon exposure to ethanol. 


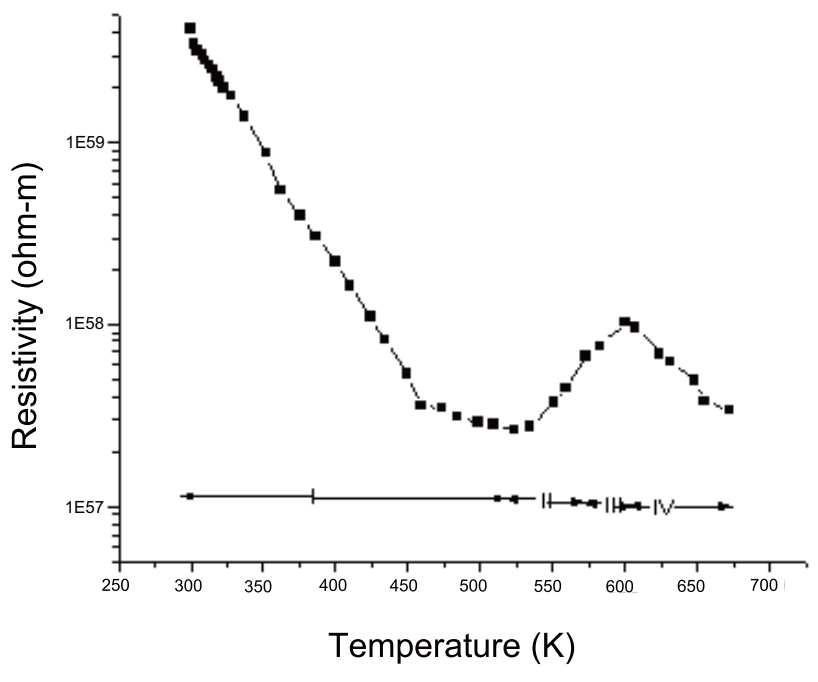

Fig. 5. Variation of resistivity of $\mathrm{SnO}_{2}$ film with temperature.

The sensing characteristics of undoped $\mathrm{SnO}_{2}$ films as a function of the operating temperature for three different concentrations, viz, 100, 300, and $500 \mathrm{ppm}$ of ethanol in air are shown in Fig. 6. The maximum sensitivity for all concentrations of ethanol was obtained at $548 \mathrm{~K}$, because most of the adsorbed oxygen species would have reacted with the $\mathrm{OH}$ group of ethanol vapor at this temperature. It has been reported that within the temperature range from 423 to $933 \mathrm{~K}$, highly active $\mathrm{O}^{-}$is the dominant species in the case of $\mathrm{SnO}_{2}{ }^{(2)}$ The major adsorbed species in our film is therefore likely to be $\mathrm{O}^{-}$. This adsorbed oxygen creates a space charge region near the film surface by extracting electrons from the material. Because ethanol is reducing by nature, it removes adsorbed $\mathrm{O}^{-}$species from the surface and reinjects the electrons back into the material, thereby reducing the resistance. The peak response at $548 \mathrm{~K}$ implies that the equilibrium density of chemisorbed $\mathrm{O}^{-}$ions is maximum at this temperature.

The ethanol-sensing mechanism of the film can be explained as follows: at first, oxygen is adsorbed on the tin dioxide layer when the film is heated in air. At lower temperatures, the surface reactions proceed too slowly to be of practical use. The adsorbed oxygen forms ionic species such as $\mathrm{O}^{2-}, \mathrm{O}_{2}^{-}$, and $\mathrm{O}^{-}$. These oxygen species when desorbed result in the increase or decrease in the resistance of the $\mathrm{SnO}_{2}$ film depending on the nature of the incoming gas. The resistance decreases if the gas is of a reducing type and increases if it is of an oxidizing type. As mentioned earlier, only $\mathrm{O}^{-}$ions will react with ethanol at the temperature range in which the sensing studies have been carried out. The reaction kinematics will proceed like this: ${ }^{(6)}$ 


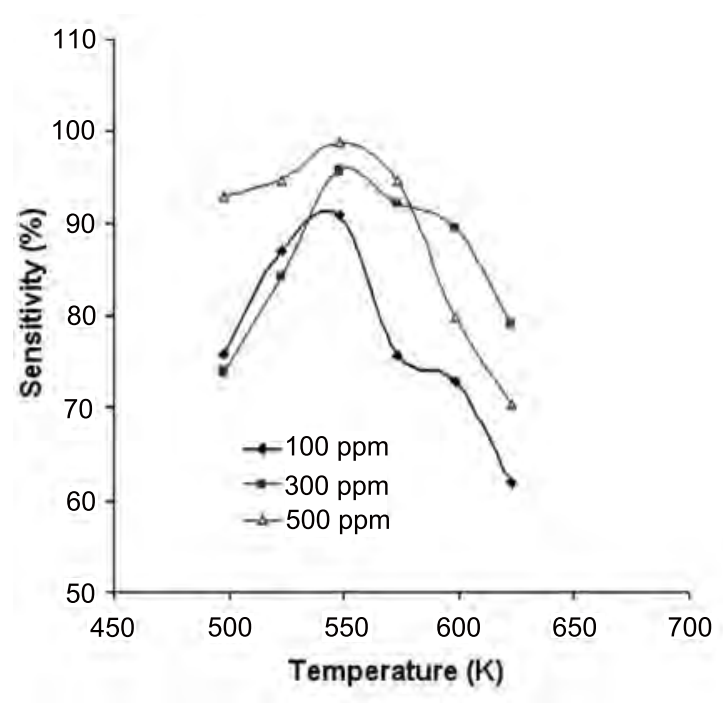

Fig. 6. Variation of sensitivity of undoped $\mathrm{SnO}_{2}$ thin film with operating temperature at different concentrations of ethanol.

$$
\begin{aligned}
\mathrm{O}_{2} \text { (gas) } & \rightleftharpoons \mathrm{O}_{2} \text { (absorbed) } \\
\mathrm{O}_{2} \text { (absorbed) }+e^{-} & \rightleftharpoons \mathrm{O}_{2}{ }^{-} \\
\mathrm{O}_{2}{ }^{-}+e^{-} & \rightleftharpoons 2 \mathrm{O}^{-}
\end{aligned}
$$

The reaction between ethanol and ionic oxygen species can take place in two different ways:

$$
\begin{aligned}
\mathrm{C}_{2} \mathrm{H}_{5} \mathrm{OH}(\text { gas })+\mathrm{O}^{-} & \rightleftharpoons \mathrm{CH}_{3} \mathrm{CHO}+\mathrm{H}_{2} \mathrm{O}+e^{-} \\
\mathrm{C}_{2} \mathrm{H}_{5} \mathrm{OH}(\text { gas }) & \rightleftharpoons \mathrm{H}+\mathrm{C}_{2} \mathrm{H}_{5} \mathrm{O}(\text { surface }) \\
\mathrm{C}_{2} \mathrm{H}_{5} \mathrm{O} & \rightleftharpoons \mathrm{H}+\mathrm{CH}_{3} \mathrm{CHO} \\
\mathrm{CH}_{3} \mathrm{CHO} \text { (gas) }+\mathrm{O}(\text { bulk }) & \left.\rightleftharpoons \mathrm{CH}_{3} \mathrm{COOH}+\mathrm{O} \text { (vacancies }\right)
\end{aligned}
$$

Figure 7 shows the sensing characteristics of tin dioxide film against concentration of ethanol in air at three different operating temperatures. It can be observed in the figure that at each operating temperature, the sensitivity increases rapidly at lower concentrations of ethanol, while it is found to increase gradually at higher concentrations of ethanol. This can be attributed to the fact that for a low concentration (100 ppm), there is a smaller surface coverage of ethanol molecules on the film, and as a result, the surface reaction proceeds slowly. With an increase in ethanol concentration to $300 \mathrm{ppm}$, the surface 


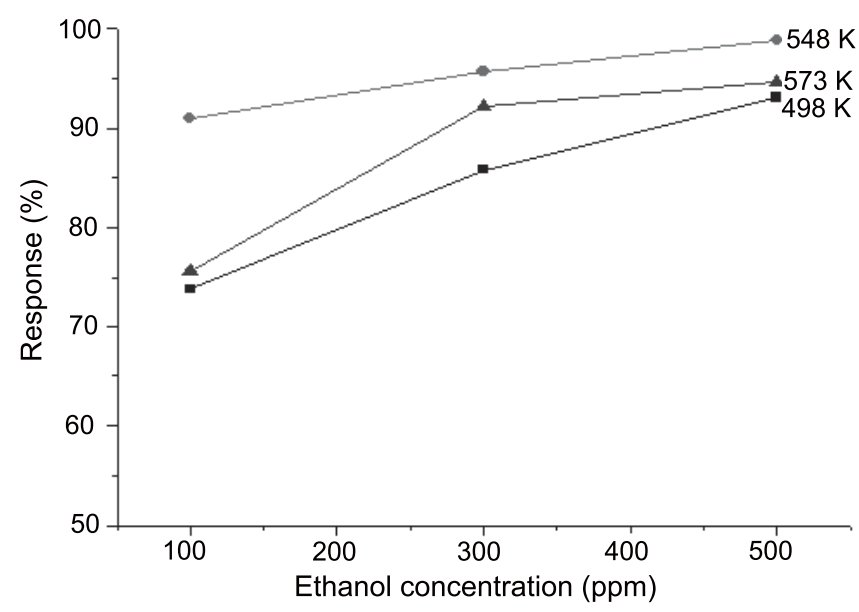

Fig. 7. Dependence of saturation sensitivity of $\mathrm{SnO}_{2}$ film on ethanol concentration at different working temperatures.

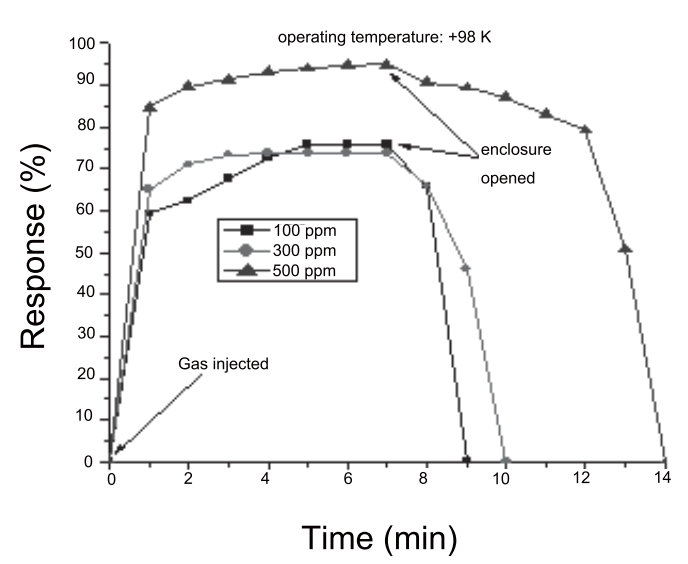

(a)

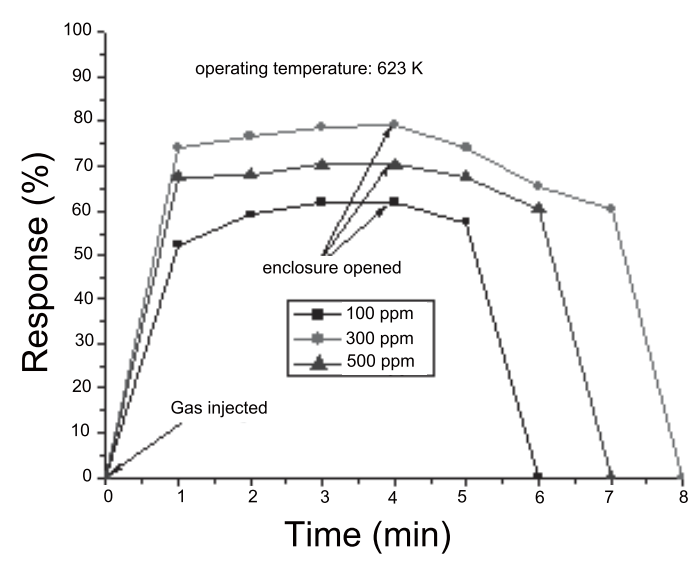

(b)

Fig. 8. Transient response characteristics of the $\mathrm{SnO}_{2}$ film at operating temperatures of (a) 498 and (b) $623 \mathrm{~K}$ for three different concentrations of ethanol.

reaction rate increases owing to a larger surface coverage of ethanol molecules, and hence, there is a rapid increase in the response. With a further increase in concentration to $500 \mathrm{ppm}$, the surface coverage of ethanol molecules on the film begins to saturate, which results in a gradual increase in response.

Figures 8(a) and 8(b) show the time dependence of the response of the film for three different concentrations $(100,300$, and $500 \mathrm{ppm})$ of ethanol at operating temperatures 
of 498 and $623 \mathrm{~K}$, respectively. It was observed that the response and recovery times were sensitively dependent on the working temperature. Both the response and recovery times were found to shorten at higher operating temperatures. This can be attributed to the fact that at higher operating temperatures, the speed of the surface reaction increases because of the sufficient thermal energy of the sensor material. However, the recovery time was found to be shorter than the response time. In this work, the average response and recovery times were found to be 5 and $2 \mathrm{~min}$, respectively.

\section{Conclusions}

$\mathrm{SnO}_{2}$ thin films of varying thicknesses have been fabricated by chemical spray pyrolysis on glass substrate. The UV-visible measurements of the samples revealed a blue shift of optical band gap with decreasing film thickness. It was observed that the response of the film was highest at $548 \mathrm{~K}$, which may be the saturation point of the redox reaction between the ethanol vapor and the adsorbed oxygen species. The sensing properties of the film were studied at a very low concentration of ethanol and it was found that the film is sensitive even at $100 \mathrm{ppm}$. The response and recovery times were found to be very small at higher operating temperatures. The results obtained by spray pyrolysis are promising for the preparation of a sensitive and low-cost ethanol sensor.

\section{Acknowledgement}

The authors are grateful to Prof. P. P. Sahay, MNIT, Allahabad, UP, India for his valuable suggestions and assistance during the conduct of this study.

\section{References}

1 T. Serin, N. Serin, S. Karadeniz, H. Sari, N. Tugluoglu and O. Pakma: J. Non-cryst. Solids. 352 (2006) 209.

2 S. Mishra, C. Ghanshyam, N. Ram, S. Singh, R. P. Bajpai and R. K. Bedi: Bull. Mater. Sci. 25 (2002) 231.

3 K. K. Makhija, A. Roy, R. M. Patel, V. B. Trivedi and H. N. Kapse: Bull. Mater. Sci. 28 (2005) 9.

4 N. G. Patel, P. D. Patel and V. S. Vaishnav: Sens. Actuators B 96 (2003)180.

5 Y. Shimizu, T. Mackawa, Y. Nakamura and M. Egashira: Sens. Actuators B 46 (1998) 163.

6 P. P. Sahay, S. Tiwari, S. Jha and M. Shamsuddin: J. Mater. Sci. 40 (2005) 4791.

7 P. P. Sahay: J. Mater. Sci. 40 (2005) 4383.

8 K. S. Shamala, L. C. S. Murthy and K. N. Rao: Indian. J. Pure Appl. Phys. 44 (2006) 867.

9 T. D. Senguttuvan, R. Rai and S. T. Lakshmikumar: J. Mater. Lett. 61 (2007) 582.

10 F. Hellegouarc'h, F. Arefi-khonsari and R. Planade: Sens. Actuators B vv73 (2001) 27.

11 P. P. Sahay, S. Tiwari and R. K. Nath: Cryst. Res. Technol. 42 (2007) 723.

12 P. Tyagi and A. G. Vedeshwar: Bull. Mater. Sci. 24 (2001) 297.

13 P. P. Sahay and R. K. Nath: Sens. Actuators B 133 (2008) 222. 